\title{
Microwave Imaging from Total Electric Field Data
}

\author{
Takashi Takenaka and Toshifumi Moriyama \\ Graduate School of Engineering \\ Nagasaki University \\ Bunkyo-machi, Nagasaki 852-8521, Japan \\ takenaka@nagasaki-u.ac.jp and t-moriya@nagasaki-u.ac.jp
}

\begin{abstract}
A novel gradient-based inverse scattering method for imaging inhomogeneous objects from time-domain data of only total electric field is presented. Unlike most inverse scattering methods, the proposed method does not require the explicit information of the incident field illuminating a region of interest. Numerical simulations demonstrate the effectiveness of the method.
\end{abstract}

\section{INTRODUCTION}

Most inverse scattering methods assume the knowledge of incident fields illuminating an region of interest [1]-[3]. We have proposed an inverse scattering approach which does not require the information of the incident field [4]. The approach uses both total electric and magnetic fields data. We have also reported reconstruction from only total electric field data using an generic algorithm (GA) as an optimization method [5]. Due to the computational complexity, GAs are not suitable for the reconstruction of complex and/or inhomogeneous objects.

In this paper, we consider the imaging of inhomogeneous objects. A gradient-based optimization is applied to the inverse scattering analysis using only total electric field data. Numerical simulations are carried out to show the effectiveness of the method.

\section{DIRECT PROBLEM}

Let us consider the scattering problem where an region of interest is illuminated by the incident field produced by an impressed electric current $\boldsymbol{J}$ which is turned on at the time $t=0$.

\section{A. Original Scattering Problem}

The total electromagnetic fields $\mathbf{v}=[\mathbf{E}, \eta \mathbf{H}]$ are the solution of the following problem:

$$
\begin{array}{ll}
\text { equation: } L \mathbf{v}(\mathbf{r}, t)=\mathbf{j}(\mathbf{r}, t), & \mathbf{r} \in \mathrm{R}^{3}, \quad t \in(0, \infty) \\
\text { initial condition: } \mathbf{v}(\mathbf{r}, 0)=\mathbf{0}, \quad \mathbf{r} \in \mathrm{R}^{3} &
\end{array}
$$

where $\mathbf{j}=\left[\begin{array}{ll}\eta \mathbf{J}, & \mathbf{0}\end{array}\right]$, and $\eta\left(=\sqrt{\mu_{0} / \varepsilon_{0}}\right)$ is the characteristic impedance of free space. The operator $L$ is defined by

$$
L \equiv \bar{A} \frac{\partial}{\partial x}+\bar{B} \frac{\partial}{\partial y}+\bar{C} \frac{\partial}{\partial z}-\bar{F} \frac{\partial}{\partial(c t)}-\bar{G}
$$

where $c\left(=1 / \sqrt{\varepsilon_{0} \mu_{0}}\right)$ is the speed of light in free space, $\bar{A}, \bar{B}$, $\bar{C}$ are $6 \times 6$ constant matrices and $\bar{F}, \bar{G}$ are $6 \times 6$ matrices contain electrical parameters (the relative permittivity $\varepsilon_{r}$, relative permeability $\mu_{r}$, and conductivity $\sigma$ ) of the medium. For more details, please refer to [6].

\section{B. Equivalent Probelm}

The original problem is equivalent to the following interior boundary value problem whose solution $\mathbf{v}^{e q}=\left[\mathbf{E}^{e q}, \eta \mathbf{H}^{e q}\right]$ is identical to $\mathbf{v}$ of the original problem (1) in the interior region $\Omega$ bounded by the observation surface $\partial \Omega$ where the total electric field $\mathbf{E}$ of the original problem is measured:

$$
\begin{aligned}
& \text { equation: } L \mathbf{v}^{e q}(\mathbf{r}, t)=\mathbf{0}, \quad \mathbf{r} \in \Omega, \quad t \in(0, \infty) \\
& \text { initial condition: } \quad \mathbf{v}^{e q}(\mathbf{r}, 0)=\mathbf{0}, \quad \mathbf{r} \in \Omega \\
& \text { boundary condition: } \\
& \qquad \hat{n} \times\left(\mathbf{E}^{e q}(\mathbf{r}, t) \times \hat{n}\right)=\hat{n} \times(\mathbf{E}(\mathbf{r}, t) \times \hat{n}), \mathbf{r} \in \partial \Omega, t \in(0, \infty) .
\end{aligned}
$$

\section{Time Reversal Problem}

By time-reversing the measured data of total electric field $\mathbf{E}$ on the observation surface $\partial \Omega$, a time reversal problem where the time-revered field $\mathbf{v}^{t r}=\left[\mathbf{E}^{t r}, \eta \mathbf{H}^{\text {tr }}\right]$ is equivalent to $\mathbf{v}$ of the original problem in the interior region $\Omega$ is set up provided that the measurement duration time $T$ is long enough for the fields in the interior region $\Omega$ to be negligibly small after the time $T$. The time-revered field $\mathbf{v}^{t r}$ is the solution of the following boundary value problem:

$$
\begin{aligned}
& \text { equation: } L \mathbf{v}^{t r}(\mathbf{r}, t)=\mathbf{0}, \quad \mathbf{r} \in \Omega, \quad t \in(0, T) \\
& \text { final condition: } \quad \mathbf{v}^{t r}(\mathbf{r}, T)=\mathbf{0}, \quad \mathbf{r} \in \Omega \\
& \text { boundary condition: } \\
& \qquad \hat{n} \times\left(\mathbf{E}^{t r}(\mathbf{r}, t) \times \hat{n}\right)=\hat{n} \times(\mathbf{E}(\mathbf{r}, t) \times \hat{n}), \mathbf{r} \in \partial \Omega, t \in(0, T)
\end{aligned}
$$

\section{INVERSE SCATTERING PROBLEM}

Let us consider the imaging problem of the electrical parameter profiles of objects of interest from the recorded data of the total electric field on the observation surface $\partial \Omega$. Note that the equivalent field and the time-reversed field is the same as the original total field generated by the impressed current $\boldsymbol{J}$ placed exterior to the surface $\partial \Omega$ if and only if the medium in $\Omega$ is the same as the original one. Using this fact, we reduce the inverse scattering problem considered here to the optimization problem minimizing the following cost functional: 


$$
Q(\mathbf{p})=\sum_{m=1}^{M} \int_{0}^{c T} \int_{\Omega} K(t)\left|\mathbf{v}_{m}^{e q}(\mathbf{p} ; \mathbf{r}, t)-\mathbf{v}_{m}^{t r}(\mathbf{p} ; \mathbf{r}, t)\right|^{2} d \mathbf{r} d(c t)
$$

where $\mathbf{p}=\left[\varepsilon_{r}(\mathbf{r}), \mu_{r}(\mathbf{r}), \eta \sigma(\mathbf{r})\right]$ is an electrical parameter vector of the object. The time factor $K(t)$ is a non-negative weighting function which takes a value of zero at $t=0$ and $T$. $M$ successive illuminations are assumed to probe the object. The vectors $\mathbf{v}_{m}^{e q}$ and $\mathbf{v}_{m}^{\text {tr }}$ are, respectively, the equivalent total field and the time-reversed total field for an estimated parameter $\mathbf{p}$ due to the $m$ th illumination.

We will minimize the functional (5) by the conjugate gradient method. Following a similar way in the reference [6], the gradient $\mathbf{g}(\mathbf{r})=\left[g_{\varepsilon}(\mathbf{r}), g_{\mu}(\mathbf{r}), g_{\sigma}(\mathbf{r})\right]$ is derived. Due to the space limitation, only $g_{\varepsilon}$ is shown:

$g_{\varepsilon}(\mathbf{p} ; \mathbf{r})$

$=\sum_{m=1}^{M} \int_{0}^{c T}\left[\mathbf{u}_{m}^{e}(\mathbf{p} ; \mathbf{r}, t) \cdot \frac{\partial \mathbf{E}_{m}^{e q}(\mathbf{p} ; \mathbf{r}, t)}{\partial(c t)}-\mathbf{w}_{m}^{e}(\mathbf{p} ; \mathbf{r}, t) \cdot \frac{\partial \mathbf{E}_{m}^{t r}(\mathbf{p} ; \mathbf{r}, t)}{\partial(c t)}\right] d(c t)$

where $\mathbf{E}_{m}^{e q}\left(\mathbf{E}_{m}^{t r}\right)$ and $\mathbf{u}_{m}^{e}\left(\mathbf{w}_{m}^{e}\right)$ are, respectively the total electric field component of the equivalent (time reversal) problem and that of its adjoint problem under the mth illumination for the object with an estimated parameter $\mathbf{p}$.

\section{NUMERICAL RESULTS}

In order to asses the proposed method, numerical simulations of reconstructing two-dimensional lossless dielectric objects are carried out. In the simulations presented here, the primary source is a $\mathrm{z}$-directed line current source $\mathbf{J}=I(t) \hat{z}$ with the time factor

$$
I(t)=\frac{d^{3}}{d t^{3}} \exp \left[-\alpha^{2}(t-\tau)^{2}\right]
$$

where $\tau=(\beta+400) \Delta t, \alpha=5.6 /(\beta \Delta t)$ and $\beta=132$ with the time step size $\Delta t=0.98(\Delta x / c \sqrt{2})=10.4 \mathrm{ps}$ and the cell size $\Delta x=\Delta y=\lambda / 10 \sqrt{4}=4.5 \mathrm{~mm}$. Here, $\lambda=90 \mathrm{~mm}$ is the wavelength in free space at the highest frequency where the source spectrum is $1 / 20$ of the maximum value. The computational domain is discretized into $180 \times 180$ cells. Twelve sources are placed on the boundary line of the $5 \lambda \times 5 \lambda$ square and are successively used to probe the cylinder. The tangential components of the total electric fields are collected on the periphery of the $4 \lambda \times 4 \lambda$ square surrounding the object. The reconstructed region is a $3 \lambda \times 3 \lambda$ square.

The first example is the reconstruction of a hollow circular cylinder of inner radius $a=0.3 \lambda$ and outer radius $b=\lambda$ (see Fig. 1(a)). It's relative permittivity has a sine-like profile with maximum value 2 :

$$
\varepsilon_{r}(r)=\left\{\begin{array}{cc}
\sin \left(\frac{\pi(r-a)}{b-a}\right)+1 & a \leq r \leq b \\
1 & \text { otherwise. }
\end{array}\right.
$$

The reconstructed result after 500 iterations is shown in Fig.1(b). This shows that the proposed method gives an accurate reconstruction.

The second example is the reconstruction of two different rectangular cylinders as shown in Fig. 2(a). The relative permittivity, size, and center location of each cylinder are $\varepsilon_{r 1}=2.0,0.7 \lambda \times 1.2 \lambda,(x=-0.5 \lambda, y=0.5 \lambda)$ and $\varepsilon_{r 2}=2.0$, $0.7 \lambda \times 0.7 \lambda, \quad(x=0.5 \lambda, y=-0.5 \lambda) \quad$, respectively. The reconstructed result after 500 iterations is shown in Fig. 2(b). Again, it can be seen that the proposed method is very effective.

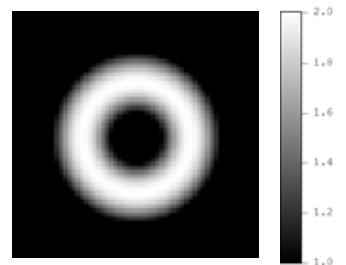

(a)

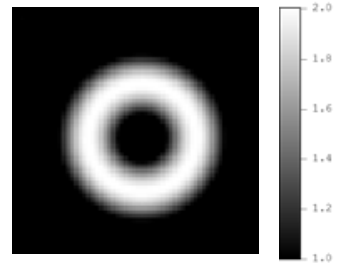

(b)
Fig. 1. Reconstruction of the relative permittivity distribution of an inhomogeneous hollow cylinder. (a) Original profile. (b) Reconstructed result of 500 iterations.

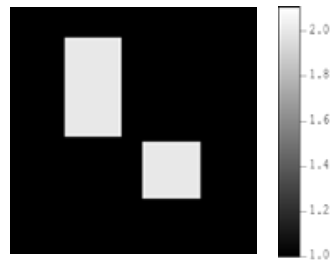

(a)

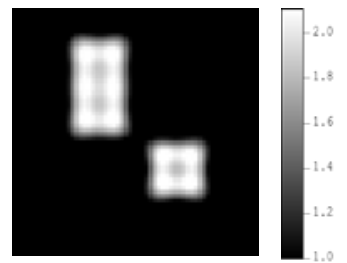

(b)
Fig. 2. Reconstruction of the relative permittivity distribution of two rectangular cylinders. (a) Original profile. (b) Reconstructed result of 500 iterations.

\section{REFERENCES}

[1] W. C. Chew and Y. M. Wang, "Reconstruction of two-dimensional permmittivity distribution using the distorted Born iterative method," IEEE Trans. Med. Imag., Vol. 9, No. 4, pp. 218-225, 1990.

[2] T. Isernia, L. Crocco, and M.D’Urso, "Newtools and series for forward and inverse scattering problems in lossy media,” IEEE Geosci. Remote Sens. Lett., vol. 1, no. 4, pp. 327-331, Oct. 2004.

[3] P. Rocca, M. Benedetti, M. Donelli, D. Franceschini, and A. Massa "Evolutionary optimization as applied to inverse problems," Inverse Problems, Vol. 25, 123003, pp. 1-41, Dec. 2009.

[4] T. Takenaka and T. Moriyama, "Inverse scattering approach based on the field equivalence principle: inversion without a priori information on incident fields, " Optics Letters, pp.3432-3434, vol. 37, no. 16, Aug. 2012.

[5] T. Takenaka, T. Moriyama, and T. Tanaka, "Image reconstruction of objects with time reversal and equivalent fields," Proc. of 2013 International Conference on Electromagnetics in Advanced Applications (ICEAA), pp.1393-1396, Torino, Italy, Sep. 2013.

[6] T. Takenaka, H. Zhou, and T. Tanaka, "Inverse scattering for a threedimensional object in the time domain,” J. Opt. Soc. Am. A, Vol.20, No.10, pp.1867-1874, Oct. 2003. 